\title{
Quantum information: Jaynes and Shannon entropies in a two-electron entangled artificial atom
}

\author{
Claudio Amovilli* \\ Dipartimento di Chimica e Chimica Industriale, Università di Pisa, Via Risorgimento 35, I-56126 Pisa, Italy \\ Norman H. March ${ }^{\dagger}$ \\ Department of Physics, University of Antwerp (RUCA), Groenenborgerlaan 171, B-2020 Antwerpen, Belgium
}

(Received 19 September 2003; revised manuscript received 28 January 2004; published 12 May 2004)

\begin{abstract}
In the context of quantum information, both Shannon entropy and the different Jaynes entropy for a twoelectron "entangled artificial" atom proposed by Moshinsky are studied here. The Jaynes entropy in this model is shown to be intimately related to the correlation kinetic energy. A generalization of the customary Shannon entropy based on the electron density is then proposed in terms of the electron pair correlation function. Both definitions behave in a similar way to the Jaynes entropy when considered as a function of the strength of the model electron-electron interaction.
\end{abstract}

DOI: 10.1103/PhysRevA.69.054302

PACS number(s): 03.67.-a

Current interest in quantum information has led us to study both Shannon and Jaynes entropies. This has been done in the context of analytical solutions for a simple example of a two-electron "entangled artificial" atom going back to Moshinsky [1]. In this model system both confining and interacting forces are of the harmonic type. The coupling constant, here called $K$, is the spring constant of the interparticle forces once the spring constant of confining forces has been set to one. It turns out that we can calculate in fact two types of entropy for this model which, following earlier usage in atomic theory, we shall term Jaynes and Shannon information entropy.

An information entropy is usually defined by the relation

$$
S=-\sum_{i} p_{i} \ln p_{i}
$$

for a discrete set of probabilities $\left(\Sigma_{i} p_{i}=1\right)$ or by the integral

$$
S=-\int d \mathbf{x} \pi(\mathbf{x}) \ln \pi(\mathbf{x})
$$

for a probability distribution depending on a given set $\mathbf{x}$ of continuous variables $\left[\int d \mathbf{x} \pi(\mathbf{x})=1\right]$. In quantum information theory, two definitions are commonly referred to, namely Jaynes and Shannon entropies. According to Eq. (1), for an electron system one can write

$$
p_{i}=\frac{n_{i}}{N}
$$

where $n_{i}$ is the occupation number of the $i$ th natural orbital (NON) and $N$ the number of electrons. The NONs correspond to the eigenvalues of the one-particle density matrix (1DM). For the Moshinsky model atom considered in this work, the $1 \mathrm{DM}$ is given by

\footnotetext{
*Electronic address: amovilli@dcci.unipi.it

${ }^{\dagger}$ Also at Oxford University, Oxford, England.
}

$$
\gamma\left(\mathbf{r} ; \mathbf{r}^{\prime}\right)=2 \int d \mathbf{r}_{2} \Psi\left(\mathbf{r}, \mathbf{r}_{2}\right) \Psi^{*}\left(\mathbf{r}^{\prime}, \mathbf{r}_{2}\right)
$$

where $\Psi\left(\mathbf{r}_{1}, \mathbf{r}_{2}\right)$ is the spin-compensated ground-state wave function. By the spectral decomposition $\gamma\left(\mathbf{r} ; \mathbf{r}^{\prime}\right)$ $=\sum_{i} n_{i} \phi_{i}(\mathbf{r}) \phi_{i}^{*}\left(\mathbf{r}^{\prime}\right)$, where the $\phi_{s}$ are the natural orbitals, the Jaynes entropy can be defined as

$$
S_{J}=-\sum_{i} \frac{n_{i}}{N} \ln \frac{n_{i}}{N}
$$

It is important to recall that for the particular case of a twoelectron system $S_{J}$ above is 0 in both the Hartree-Fock (HF) and the noninteracting limit (no interparticle interactions), $\Psi\left(\mathbf{r}_{1}, \mathbf{r}_{2}\right)$ being a simple product of orbitals in these particular situations.

In order to diagonalize the 1DM to get the Jaynes entropy (5) we start from $[2,3]$

$$
\gamma\left(\mathbf{r}_{1}, \mathbf{r}_{2}\right)=\mathcal{N}^{2} \exp \left[-A\left(r_{1}^{2}+r_{2}^{2}\right)-B r_{12}^{2}\right]
$$

where

$$
\mathcal{N}=\sqrt{2}\left(\frac{2 \alpha-1}{\pi \alpha}\right)^{3 / 4}, \quad A=\frac{2 \alpha-1}{2 \alpha}, \quad B=\frac{(\alpha-1)^{2}}{4 \alpha},
$$

in which $\alpha=(1 / 2)[1+\sqrt{1+2 K}]$. The above $1 \mathrm{DM}$ can only be diagonalized by numerical procedures but some preliminary analytical work is helpful to define a good basis set for the calculation of natural orbitals. Developing $r_{12}^{2}$ in (6) and by power expanding the exponential factor containing the scalar product $\vec{r}_{1} \cdot \vec{r}_{2}$, one gets

$$
\begin{aligned}
\gamma\left(\mathbf{r}_{1}, \mathbf{r}_{2}\right)= & \sum_{i j k} \frac{(2 B)^{i+j+k}}{i ! j ! k !} \times\left\{\mathcal{N} x_{1}^{i} y_{1}^{j} z_{1}^{k} \exp \left[-(A+B) r_{1}^{2}\right]\right\} \\
& \times\left\{\mathcal{N} x_{2}^{i} y_{2}^{j} z_{2}^{k} \exp \left[-(A+B) r_{2}^{2}\right]\right\}
\end{aligned}
$$

which suggests that one expand the natural orbitals in terms of the basis functions 


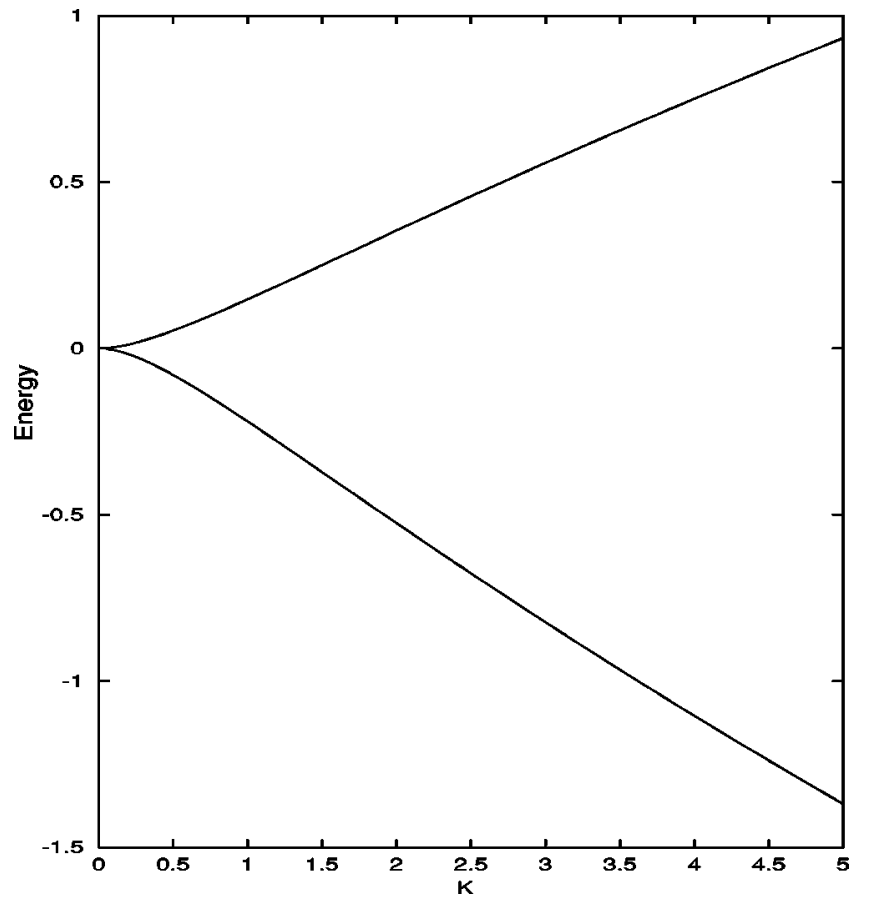

FIG. 1. Correlation kinetic energy contributions $T_{W}^{(c o r r)}$ (lower curve) and $6 B$ (upper curve) of Eq. (11) against the interparticle spring constant $K$. Data are in atomic units.

$$
\varphi_{i j k}=x^{i} y^{j} z^{k} \exp \left[-(A+B) r^{2}\right] .
$$

As one can see from Eq. (8), although this equation cannot be seen as a spectral decomposition because of the nonorthogonality of the functions $\varphi_{i j k}$ above, the constant $B$ plays an important role in the definition of the spectrum of occupation numbers. We remark here that $B$ is $1 / 6$ of the difference between the exact kinetic energy and the von Weizsäcker term, an important fraction of the correlation kinetic energy. Following the Löwdin definition, the correlation kinetic energy is given by

$$
T_{\text {corr }}=T_{e x}-T_{H F},
$$

where $T_{e x}$ and $T_{H F}$ are, respectively, the exact and HF total kinetic energies. Recalling the Weizsäcker definition of kinetic energy, namely $T_{W}=|\vec{\nabla} \rho|^{2} / 8 \rho$, one can decompose the above correlation kinetic energy into the following contributions:

$$
T_{\text {corr }}=\frac{|\vec{\nabla} \rho|^{2}}{8 \rho}-\frac{\left|\vec{\nabla} \rho_{H F}\right|^{2}}{8 \rho_{H F}}+6 B=T_{W}^{c o r r}+6 B .
$$

A plot of the two terms $T_{W}^{c o r r}$ and $6 B$, against the spring constant of the interparticle interaction forces, is shown in Fig. 1. The exact kinetic energy is, in this model from the virial theorem, one-half of the exact (in principle observable: say in a quantum dot if it could also be manufactured for two Fermions interacting with harmonic interparticle force) ground-state total energy.

Next we turn to the second type of entropy considered in this work. Although the Shannon information entropy is commonly defined in terms of the density $\rho(\mathbf{r})$ of a many particle system, here we prefer to extend such definition also to the two-particle density function $\rho_{2}\left(\mathbf{r}_{1}, \mathbf{r}_{2}\right)$. In particular, according to (2) we define the following two types of Shannon entropies:

$$
S_{S 1}=-\int d \mathbf{r} \pi_{1}(\mathbf{r}) \ln \pi_{1}(\mathbf{r})
$$

and

$$
S_{S 2}=-\int d \mathbf{r}_{1} d \mathbf{r}_{2} \pi_{2}\left(\mathbf{r}_{1}, \mathbf{r}_{2}\right) \ln \pi_{2}\left(\mathbf{r}_{1}, \mathbf{r}_{2}\right)
$$

where

$$
\pi_{2}\left(\mathbf{r}_{1}, \mathbf{r}_{2}\right)=\left|\Psi\left(\mathbf{r}_{1}, \mathbf{r}_{2}\right)\right|^{2}
$$

and

$$
\pi_{1}(\mathbf{r})=\int d \mathbf{r}_{2} \pi_{2}\left(\mathbf{r}, \mathbf{r}_{2}\right)
$$

$\pi_{1}$ and $\pi_{2}$ are, respectively, proportional to $\rho(\mathbf{r})$ and $\rho_{2}\left(\mathbf{r}_{1}, \mathbf{r}_{2}\right)$. In the textbooks of information theory, the entropies defined in terms of a joint probability density function, like that of Eq. (13), are called joint differential entropies (see, for example, [4]). In this case, both $S_{S 1}$ and $S_{S 2}$ do not go to zero at the noninteracting limit, so, for comparison purposes with the Jaynes entropy, it is preferable to compute the correlation Shannon entropies by writing

$$
\Delta S_{S j}^{(c o r r)}=S_{S j}-S_{S j}^{(H F)},
$$

where the $S_{S j}^{(H F)}$ are calculated by using $\Psi_{H F}\left(\mathbf{r}_{1}, \mathbf{r}_{2}\right)$ $=\phi_{H F}\left(\mathbf{r}_{1}\right) \phi_{H F}\left(\mathbf{r}_{2}\right)$. For the two-electron Moshinsky model atom, the analytical forms of the exact and HF wave functions are known [1,5] and allow the analytical derivation of both the correlation entropies of Eq. (16). After simple calculation, one readily gets

$$
\Delta S_{S 1}^{(c o r r)}=-\frac{3}{2} \ln \left[\frac{2}{(1+\sqrt{1+2 K})} \sqrt{\frac{1+2 K}{1+K}}\right]
$$

and

$$
\Delta S_{S 2}^{(c o r r)}=-\frac{3}{2} \ln \left[\frac{\sqrt{1+2 K}}{1+K}\right] .
$$

Here we give the "unpaired" Shannon entropy from the Hartree-Fock theory, namely $S_{S 1}^{(H F)}$, for comparison with the "excess entanglement" given by Eq. (17). The role of the excess entanglement in quantum information theory has been discussed in a recent paper by Shi [6]. $S_{S 1}^{(H F)}$ is recorded below in Eq. (19) and is plotted also in Fig. 2,

$$
S_{S 1}^{(H F)}=\frac{3}{2}(1+\ln \pi)-\frac{3}{4} \ln (1+K) .
$$

$\Delta S_{S 2}^{(\text {corr })}$ contains most of the informations about the entanglement of the two electrons, being more directly related to the exact wave function. It is very interesting to notice that $\Delta S_{S 1}^{(c o r r)}$ and $\Delta S_{S 2}^{(\text {corr })}$ are simply related by the difference 


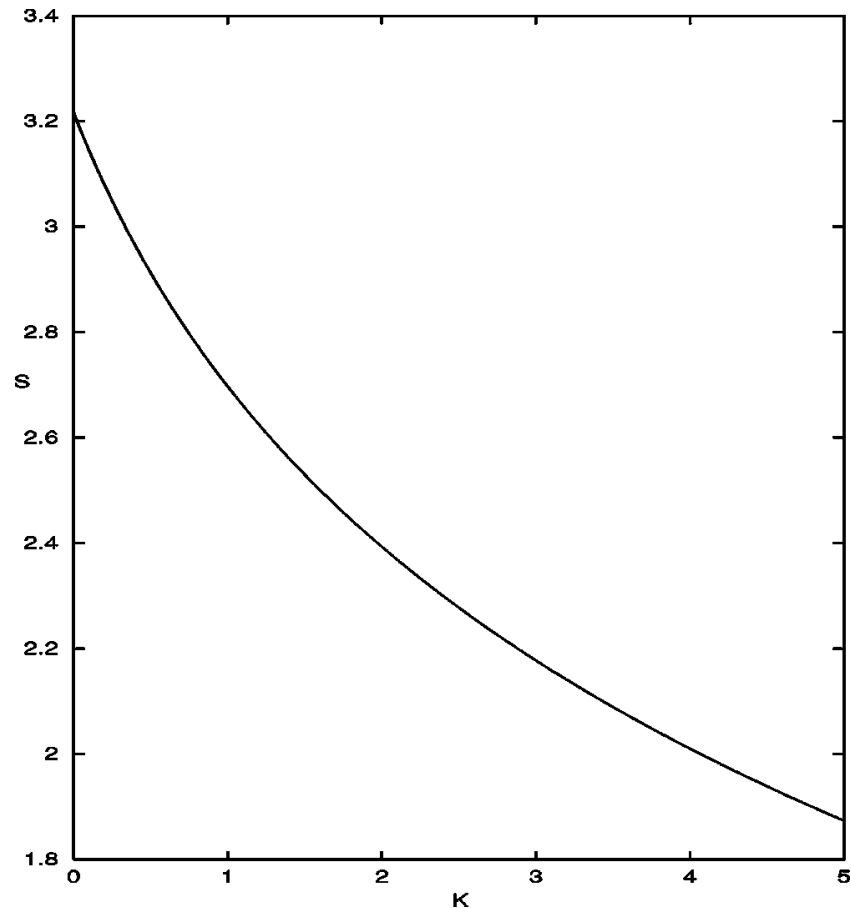

FIG. 2. Plot of Shannon entropy defined in Eq. (12) got from Hartree-Fock density against the interparticle spring constant $K$. Data are in atomic units.

$$
\Delta S_{S 1}^{(c o r r)}-\Delta S_{S 2}^{(c o r r)}=-\frac{3}{2} \ln \left[\frac{2 \sqrt{1+K}}{1+\sqrt{1+2 K}}\right],
$$

which is constant in the strongly correlated limit $(K \rightarrow \infty)$. Finally in Fig. 3 we show how Jaynes and Shannon correla-

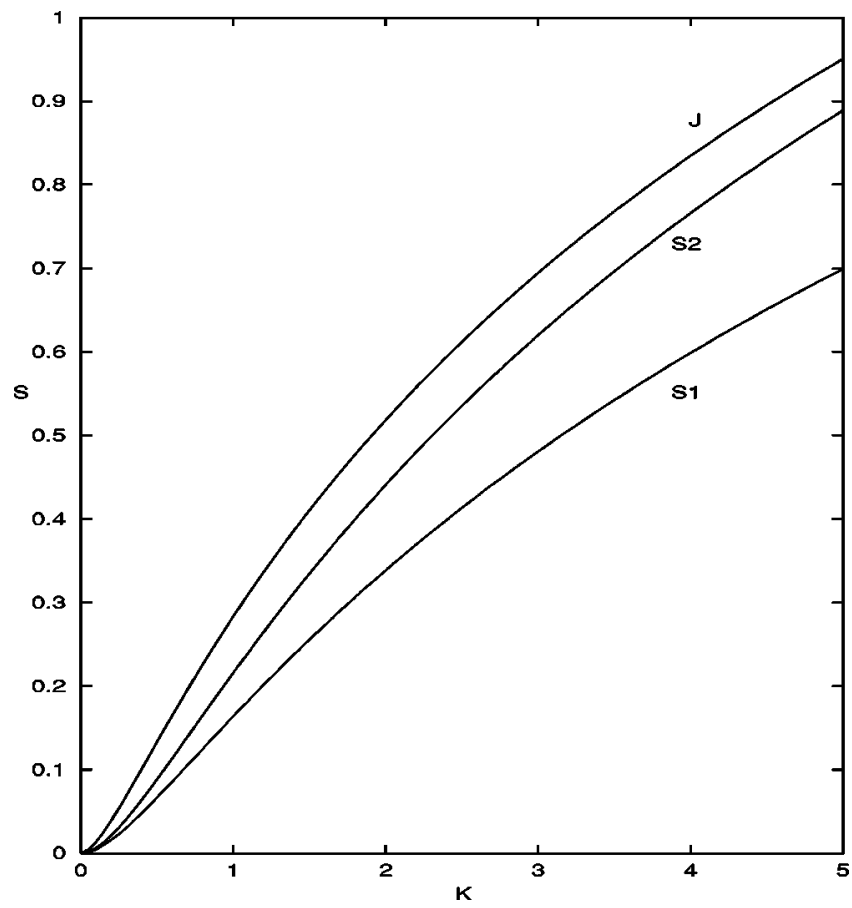

FIG. 3. Plot of Jaynes entropy $(J)$ and of correlation Shannon entropies ( $S 1$ and $S 2$ ) defined in Eq. (16) against the interparticle spring constant $K$. Data are in atomic units.

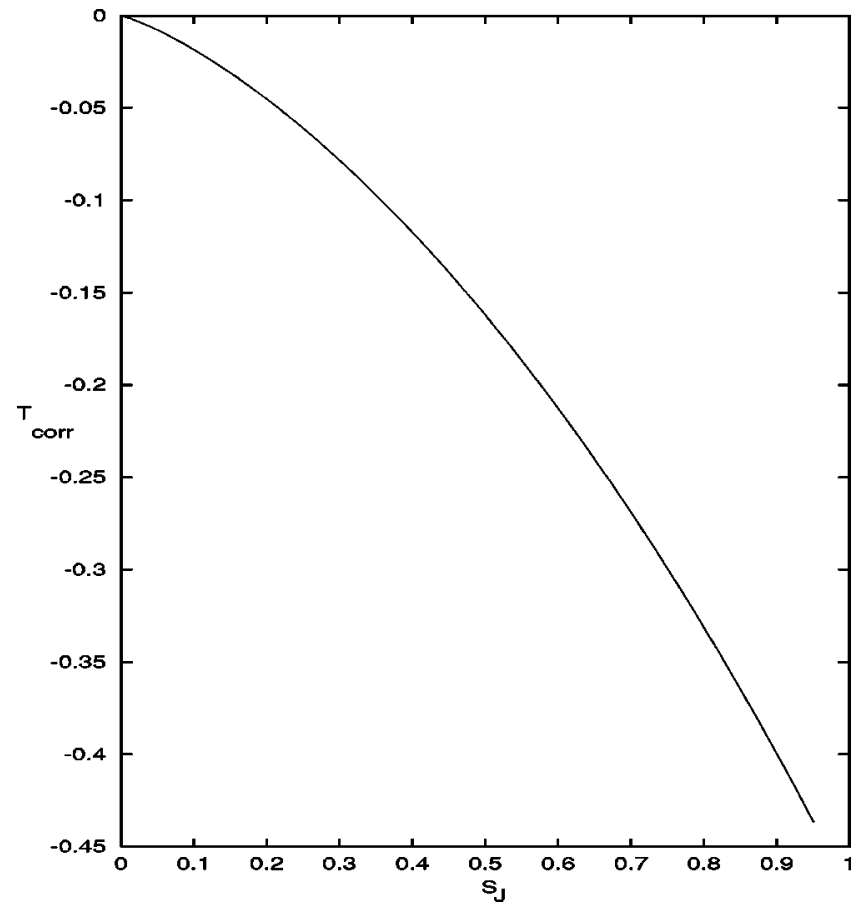

FIG. 4. Plot of the correlation kinetic energy against Jaynes entropy. Data are in atomic units.

tion entropies vary with the interparticle interaction strength $K$. Figure 3 shows clearly that also the Jaynes entropy is intimately related to $\Delta S_{S 2}^{(\text {corr })}$ and, consequently, that the deviation from the idempotency of $\gamma / 2$ is a result of the entanglement.

Finally, a simple analysis of Figs. 1 and 3 leads to the conclusion that the correlation kinetic energy and the two contributions of Eq. (11) contain the information entropy in either Jaynes or Shannon definitions given in the present work. By way of example, a plot of the correlation kinetic energy against the Jaynes information entropy is shown in Fig. 4. For this model system, the correlation kinetic energy from the virial theorem is $1 / 2$ of the correlation energy.

In conclusion, we should note that by numerical procedures, Esquivel et al. [7] have argued that the Jaynes entropy is correlated with $E_{c o r r}$, the correlation energy, and we have shown that correlation here specifically for the correlation kinetic energy of the Moshinsky atom. Turning to the Shannon entropy, we have proposed in Eq. (13) an alternative definition in terms of the electronic pair correlation function. Figure 3 shows that for the "entangled artificial" atom considered throughout this Brief Report, this definition has similarity of shape, as a function of electron-electron interaction strength, with the customary definition (12). Neither Shannon form differs qualitatively from the Jaynes entropy in the present model.

N.H.M. wishes to acknowledge that his contribution to this study was brought to fruition during a visit to ICTP, Trieste. It is a pleasure to thank Professor V. E. Kravtsov for the stimulating atmosphere provided and for generous hospitality. 
[1] M. Moshinsky, Am. J. Phys. 36, 52 (1968).

[2] C. Amovilli and N. H. March, Phys. Rev. A 67, 022509 (2003).

[3] A. Holas, I. A. Howard, and N. H. March, Phys. Lett. A 310, 451 (2003).

[4] T. M. Cover and J. A. Thomas, Elements of Information
Theory (Wiley-Interscience, New York, 1991).

[5] L. E. Ballentine, Quantum Mechanics (Prentice-Hall, London, 1972).

[6] Y. Shi, Phys. Rev. A 67, 024301 (2003).

[7] R. O. Esquivel, A. L. Rodriguez, R. P. Sagar, M. Ho, and V. H. Smith, Jr., Phys. Rev. A 54, 259 (1996). 